\title{
The Explicit Pure Vector Superfield in Gauge Theories
}

\author{
Edouard (Edward) B. Manoukian \\ The Institute for Fundamental Study, Naresuan University, Phitsanulok, Thailand \\ Email: manoukian eb@hotmail.com
}

Received May 7, 2012; revised June 1, 2012; accepted June 30, 2012

\begin{abstract}
An explicit expression of the pure vector superfield is derived in gauge theories in the Wess-Zumino gauge. A pure vector superfield means that the theta independent part of the superfield transforms as a Lorentz vector. This is to be contrasted with the so-called general scalar superfield, whose theta independent part is a scalar, as well as with the known spinor superfield, whose theta independent part is a spinor, which both contain a vector field. In contrast to the latter two superfields, the action of supersymmetric gauge theories follows directly from the theory of a pure vector superfield from a so-called $\mathcal{D}$-term. As the construction of a supersymmetric gauge theory of Yang-Mills vector Bosons, is more naturally generated out of a pure vector supersfield and not of a scalar or a spinor superfield, the importance of a pure vector superfield cannot be overemphasized.
\end{abstract}

Keywords: Pure Vector Superfield; Supersymmetry; Wess-Zumino Gauge

\section{Introduction}

We derive an explicit expression for the pure vector superfield in gauge theories in the Wess-Zumino gauge from which the supersymmetric action is directly obtained from a so-called $\mathcal{D}$-term. By a pure vector superfield, it is meant that its theta independent part transforms as a Lorentz vector. The pure vector superfield is not to be confused with the well known (scalar)-vector superfield [1-4] obtained by imposing a reality condition on the general scalar superfield, whose theta independent part is a scalar, and neither is to be confused with the well known spinor superfield [1-4], whose theta independent part is a spinor, both containing a vector field, and the supersymmetric action is obtained from the latter from a so-called $\mathcal{F}$-term. Although the derivation is somehow tedious, the theta dependent part of the pure vector superfield turns out to be not complicated.

\section{The Pure Vector Superfield: Its Explicit Expression}

In the celebrated Wess-Zumino gauge, and in a four component representation, the (scalar)-vector superfield takes the form $[5,6]$

$$
\begin{aligned}
\mathcal{V}(x, \theta)= & \frac{1}{4} \bar{\theta} \gamma^{5} \gamma^{\mu} \theta V_{\mu}(x)-\frac{\mathrm{i}}{2 \sqrt{2}} \bar{\theta} \gamma^{5} \theta \bar{\theta} \chi(x) \\
& -\frac{1}{16}\left(\bar{\theta} \gamma^{5} \theta\right)^{2} K(x),
\end{aligned}
$$

with the following residual gauge transformation

$$
\begin{aligned}
& \exp (-2 g \mathcal{V}) \rightarrow \exp \left(\operatorname{ig} \Lambda^{\dagger}\right) \exp (-2 g \mathcal{V}) \exp (-\operatorname{ig} \Lambda) \\
& \equiv \exp \left(-2 g \mathcal{V}^{\prime}\right),
\end{aligned}
$$

where the gauge function $\Lambda(x, \theta)$ is given by

$$
\begin{aligned}
& \Lambda(x, \theta)=a(x)-\frac{\mathrm{i}}{4} \bar{\theta} \gamma^{5} \gamma^{\mu} \theta \partial_{\mu} a(x)-\frac{1}{32}\left(\bar{\theta} \gamma^{5} \theta\right)^{2} \partial^{2} a(x), \\
& a=\text { Re } a .
\end{aligned}
$$

One may define a pure vector superfield $[5,6]$ as follows

$$
\mathcal{V}^{\mu}=-\frac{1}{2 \mathrm{~g}}\left(\mathcal{C} \gamma^{\mu}\right)_{a b} D_{a}^{\mathrm{R}} \mathrm{e}^{2 \mathrm{~g} \mathcal{V}} D_{b}^{\mathrm{L}} \mathrm{e}^{-2 \mathrm{~g} \mathcal{\nu}},
$$

where $\mathcal{C}$ is the charge conjugation matrix in the chiral representation. Under the supergauge transformation Equation (2),

$$
\mathcal{V}^{\mu} \rightarrow-\frac{1}{2 \mathrm{~g}}\left(\mathcal{C} \gamma^{\mu}\right)_{a b} D_{a}^{\mathrm{R}} \mathrm{e}^{\mathrm{ig} \Lambda} \mathrm{e}^{2 \mathrm{~g} \mathcal{V}} \mathrm{e}^{-\mathrm{ig} \Lambda^{\dagger}} D_{b}^{\mathrm{L}} \mathrm{e}^{\mathrm{ig} \Lambda^{\dagger}} \mathrm{e}^{-2 \mathrm{~g} \mathcal{V}} \mathrm{e}^{-\mathrm{ig} \Lambda},
$$

where we recall that $\Lambda$ is left-chiral and hence $\Lambda^{\dagger}$ is right-chiral. Accordingly, they are, respectively, annihilated by the supercovariant derivatives

$$
D^{\mathrm{R} / \mathrm{L}} \equiv\left(1 \mp \gamma^{5} / 2\right) D,
$$

where $D=\partial / \partial \bar{\theta}-(\mathrm{i} / 2)\left(\gamma^{\mu} \theta\right) \partial_{\mu}$. That is,

$$
D^{\mathrm{R}} \mathrm{e}^{\mathrm{i} g \Lambda}=\mathrm{e}^{\mathrm{i} g \Lambda} D^{\mathrm{R}}, \quad D^{\mathrm{L}} \mathrm{e}^{\mathrm{i} g \Lambda^{\dagger}}=\mathrm{e}^{\mathrm{i} g \Lambda^{\dagger}} D^{\mathrm{L}} .
$$


We may rewrite the transformation rule in Equation (5) as

$$
\begin{aligned}
& \mathcal{V}^{\mu} \rightarrow-\frac{1}{2 \mathrm{~g}} \mathrm{e}^{\mathrm{ig} \Lambda}\left(\mathcal{C} \gamma^{\mu}\right)_{a b} D_{a}^{\mathrm{R}} \mathrm{e}^{2 \mathrm{~g} \mathcal{V}} \times\left(D_{b}^{\mathrm{L}} \mathrm{e}^{-2 \mathrm{~g} \mathcal{}} \mathrm{e}^{-\mathrm{ig} \Lambda}\right) \\
& =-\frac{1}{2 \mathrm{~g}} \mathrm{e}^{\mathrm{i} \mathrm{g} \Lambda}\left(\mathcal{C} \gamma^{\mu}\right)_{a b} D_{a}^{\mathrm{R}} \mathrm{e}^{2 \mathrm{~g} \mathcal{V}} \times\left(D_{b}^{\mathrm{L}} \mathrm{e}^{-2 \mathrm{~g} \mathcal{V}}\right) \mathrm{e}^{-\mathrm{ig} \Lambda} \\
& -\frac{1}{2 \mathrm{~g}} \mathrm{e}^{\mathrm{ig} \Lambda}\left(\mathcal{C} \gamma^{\mu}\right)_{a b}\left(D_{a}^{\mathrm{R}} D_{b}^{\mathrm{L}} \mathrm{e}^{-\mathrm{i} g \Lambda}\right) .
\end{aligned}
$$

Due to the first equality in Equation (6), we may replace the product $D_{a}^{\mathrm{R}} D_{b}^{\mathrm{L}}$ in the second term on the extreme right-hand side of Equation (7) by their anticommutator. This anti-commutator may be obtained from $\left\{D_{a}, D_{b}\right\}=-\mathrm{i}\left(\gamma^{\mu} \mathcal{C}\right)_{a b} \partial_{\mu}$ by multiplying it by

$$
\left(1-\gamma^{5}\right)_{a a^{\prime}}\left(1+\gamma^{5}\right)_{b b^{\prime}} / 4
$$

leading to

$$
\mathcal{V}^{\mu} \rightarrow \mathrm{e}^{\mathrm{ig} \Lambda} \mathcal{V}^{\mu} \mathrm{e}^{-\mathrm{ig} \Lambda}+\frac{\mathrm{i}}{\mathrm{g}} \mathrm{e}^{\mathrm{ig} \Lambda} \partial_{\mu} \mathrm{e}^{-\mathrm{ig} \Lambda},
$$

and showing that it transforms as a non-abelian gauge field.

$$
\begin{aligned}
& \text { Using the relations }\left\{\gamma^{5}, \gamma^{\mu}\right\}=0,\left[\gamma^{5}, \mathcal{C}\right]=0, \\
& \qquad\left(\left(1+\gamma^{5}\right) / 2\right)^{2}=\left(1+\gamma^{5}\right) / 2
\end{aligned}
$$

Equation (4) may be equivalently re-expressed as

$$
\mathcal{V}^{\mu}=-\frac{1}{2 \mathrm{~g}}\left(\mathcal{C} \gamma^{\mu} \frac{1+\gamma^{5}}{2}\right)_{a b} D_{a} \mathrm{e}^{2 \mathrm{~g} v} D_{b} \mathrm{e}^{-2 \mathrm{~g} \nu}
$$

In the Wess-Zumino gauge,

$$
\begin{aligned}
\mathrm{e}^{-2 \mathrm{~g} \nu}= & 1-\frac{\mathrm{g}}{2} \bar{\theta} \gamma^{5} \gamma^{\mu} \theta V_{\mu} \\
& +\frac{\mathrm{ig}}{\sqrt{2}} \bar{\theta} \gamma^{5} \theta \bar{\theta} \chi+\frac{\mathrm{g}}{8}\left(\bar{\theta} \gamma^{5} \theta\right)^{2}\left[K+\mathrm{g} V^{\nu} V_{\nu}\right]
\end{aligned}
$$

Applying the supercovariant derivative $D_{b}$ to it and using, in the process, the expansion of the product

$$
\theta_{a} \bar{\theta}_{b}=-(1 / 4 /)\left[\delta_{a b} \bar{\theta} \theta+\gamma_{a b}^{5} \bar{\theta} \gamma^{5} \theta+\left(\gamma^{5} \gamma_{\mu}\right)_{a b} \bar{\theta} \gamma^{5} \gamma^{\mu} \theta\right],
$$

together with the orthogonality relations between the product of any of two of $\bar{\theta} \gamma^{5} \theta, \bar{\theta} \theta, \bar{\theta} \gamma^{5} \gamma^{\mu} \theta$, give

$$
\begin{aligned}
& \frac{1}{\mathrm{~g}} D_{b} \mathrm{e}^{-2 \mathrm{~g} v} \\
= & -\left(\gamma^{5} \gamma^{\mu} \theta\right)_{b} V_{\mu}+\frac{\mathrm{i}}{4} \bar{\theta} \gamma^{5} \gamma^{\mu} \theta\left(\gamma^{\sigma} \theta\right)_{b} \partial_{\sigma} V_{\mu} \\
& -\frac{\mathrm{i}}{2 \sqrt{2}}\left\{\bar{\theta} \theta\left(\gamma^{5} \chi\right)_{b}-\bar{\theta} \gamma^{5} \theta \chi_{b}+\bar{\theta} \gamma^{5} \gamma_{\lambda} \theta\left(\gamma^{\lambda} \chi\right)_{b}\right\} \\
& -\frac{1}{8 \sqrt{2}}\left(\bar{\theta} \gamma^{5} \theta\right)^{2}\left(\gamma^{\sigma} \gamma^{5} \partial_{\sigma} \chi\right)_{b} \\
+ & +\frac{1}{2} \bar{\theta} \gamma^{5} \theta\left(\gamma^{5} \theta\right)_{b}\left[K+\mathrm{g} V^{v} V_{v}\right] .
\end{aligned}
$$

Multiplying the latter equation by

$$
\left[\mathcal{C} \gamma^{\rho}\left(1+\gamma^{5}\right) / 2\right]_{a b} \exp (2 \mathrm{~g} \mathcal{V}),
$$

from the left, leads to

$$
\begin{aligned}
& \frac{1}{\mathrm{~g}}\left(\mathcal{C} \gamma^{\rho} \frac{1+\gamma^{5}}{2}\right)_{a b} \mathrm{e}^{2 \mathrm{~g} \nu} D_{b} \mathrm{e}^{-2 \mathrm{~g} \nu} \\
= & -\left(\mathcal{C} \gamma^{\rho} \frac{1+\gamma^{5}}{2} \gamma^{\mu} \theta\right)_{a} V_{\mu} \\
& -\frac{\mathrm{i}}{4} \bar{\theta} \gamma^{5} \theta\left(\mathcal{C} \gamma^{\rho} \frac{1+\gamma^{5}}{2} \gamma^{\sigma} \gamma^{\mu} \theta\right)_{a} \partial_{\sigma} V_{\mu} \\
& -\frac{\mathrm{i}}{2 \sqrt{2}}\left\{\left(\bar{\theta} \theta-\bar{\theta} \gamma^{5} \theta\right)\left(\mathcal{C} \gamma^{\rho} \frac{1+\gamma^{5}}{2} \chi\right)_{a}\right. \\
& \left.\left.+\frac{1}{8 \sqrt{2} \gamma^{5} \gamma_{\lambda} \theta\left(\mathcal{C} \gamma^{\rho}\right.} \frac{1+\gamma^{5}}{2} \gamma^{\lambda} \theta\right)_{a}\right\} \\
& \left.\left.+\frac{1}{2} \bar{\theta} \gamma^{5} \theta\right)^{2} \theta\left(\mathcal{C} \gamma^{\rho} \frac{1+\gamma^{5}}{2} \gamma^{\sigma} \gamma^{5} \partial_{\sigma} \chi\right)_{a} \frac{1+\gamma^{5}}{2} \theta\right)_{a}\left[K+\mathrm{g} V^{\nu} V_{\nu}\right] \\
& +\frac{\mathrm{g}}{2} \bar{\theta} \gamma^{5} \theta\left(\mathcal{C} \gamma^{\rho} \frac{1+\gamma^{5}}{2} \gamma^{\sigma} \gamma^{\mu} \theta\right)_{a} V_{\mu} V_{\sigma} \\
& -\frac{\mathrm{ig}}{4 \sqrt{2}}\left(\bar{\theta} \gamma^{5} \theta\right)^{2}\left(\mathcal{C} \gamma^{\rho} \frac{1+\gamma^{5}}{2} \gamma^{\sigma}\left[V_{\sigma} \chi-\chi V_{\sigma}\right]\right)_{a}
\end{aligned}
$$

Now we apply $-D_{a} / 2$ to the above equation, and use, in the process, the following properties,

$$
\begin{aligned}
& \theta_{a} \mathcal{C}_{a b}=\bar{\theta}_{b}, \\
& \left(\gamma^{5} \theta\right)_{a} \mathcal{C}_{a b}=\left(\bar{\theta} \gamma^{5}\right)_{b}, \\
& \left(\gamma^{\lambda} \theta\right)_{a} \mathcal{C}_{a b}=-\left(\bar{\theta} \gamma^{\lambda}\right)_{b}, \\
& \left(\gamma^{5} \gamma^{\lambda} \theta\right)_{a} \mathcal{C}_{a b}=-\left(\bar{\theta} \gamma^{\lambda} \gamma^{5}\right)_{b},
\end{aligned}
$$

to obtain

$$
\begin{aligned}
\mathcal{V}^{\rho}(x, \theta)= & V^{\rho}(x)+\frac{\mathrm{i}}{\sqrt{2}} \bar{\theta} \gamma^{\rho} \chi(x)-\bar{\theta} \gamma^{5} \gamma_{\lambda} \theta A^{\lambda \rho}(x) \\
& -\bar{\theta} \gamma^{5} \theta \bar{\theta} B^{\rho}(x)-\left(\bar{\theta} \gamma^{5} \theta\right)^{2} C^{\rho}(x),
\end{aligned}
$$

where

$$
\begin{aligned}
& A^{\lambda \rho} \\
= & \frac{\mathrm{i}}{16} \operatorname{Tr}\left[\left(\gamma^{\sigma} \gamma^{\rho} \gamma^{\mu} \gamma^{\lambda} \frac{1+\gamma^{5}}{2}\right)+\left(\gamma^{\rho} \gamma^{\sigma} \gamma^{\mu} \gamma^{\lambda} \frac{1-\gamma^{5}}{2}\right)\right] \partial_{\sigma} V_{\mu} \\
& -\frac{\mathrm{g}}{8} \operatorname{Tr}\left[\gamma^{\rho} \gamma^{\sigma} \gamma^{\mu} \gamma^{\lambda} \frac{1-\gamma^{5}}{2}\right] V_{\mu} V_{\sigma}+\frac{1}{4} \eta^{\rho \lambda}\left[K+\mathrm{g} V^{\nu} V_{v}\right],
\end{aligned}
$$




$$
\begin{aligned}
B^{\rho}= & \frac{1}{2 \sqrt{2}}\left(\eta^{\rho \sigma}+\frac{1}{2} \gamma^{5} \gamma^{\rho} \gamma^{\sigma}\right) \partial_{\sigma} \chi \\
& +\frac{\mathrm{ig}}{2 \sqrt{2}} \gamma^{\rho} \gamma^{\sigma} \frac{1-\gamma^{5}}{2}\left(V_{\sigma} \chi-\chi V_{\sigma}\right), \\
C^{\rho}= & -\frac{\mathrm{i}}{16} \partial^{\rho}\left[K+\mathrm{g} V^{\nu} V_{v}\right] \\
& +\frac{\mathrm{ig}}{32} \operatorname{Tr}\left[\gamma^{\lambda} \gamma^{\rho} \gamma^{\sigma} \gamma^{\mu} \frac{1+\gamma^{5}}{2}\right] \partial_{\lambda}\left(V_{\mu} V_{\sigma}\right) \\
& +\frac{1}{64} \operatorname{Tr}\left[\gamma^{\lambda} \gamma^{\rho} \gamma^{\sigma} \gamma^{\mu} \frac{1+\gamma^{5}}{2}\right] \partial_{\lambda} \partial_{\sigma} V_{\mu} .
\end{aligned}
$$

The identities

$$
\begin{aligned}
& \operatorname{Tr}\left[\gamma^{\sigma} \gamma^{\rho} \gamma^{\mu} \gamma^{\lambda}\right]=4\left(\rho^{\sigma \rho} \rho^{\mu \lambda}-\rho^{\sigma \mu} \rho^{\rho \lambda}+\rho^{\sigma \lambda} \rho^{\rho \mu}\right), \\
& \operatorname{Tr}\left[\gamma^{\sigma} \gamma^{\rho} \gamma^{\mu} \gamma^{\lambda} \gamma^{5}\right]=-\mathrm{i} 4 \varepsilon^{\sigma \rho \mu \lambda},
\end{aligned}
$$

and $\varepsilon^{\lambda \rho \sigma \mu} \partial_{\lambda} \partial_{\sigma} V_{\mu}=0$, lead to the following expressions for $A^{\rho \lambda}$, and $C^{\rho}$,

$$
\begin{aligned}
A^{\lambda \rho}(x)= & \frac{\mathrm{i}}{4} \partial^{\lambda} V^{\rho}(x)-\frac{\mathrm{i}}{4} G^{\lambda \rho}(x) \\
& -\frac{1}{8} \varepsilon^{\rho \sigma \mu \lambda} G_{\sigma \mu}(x)+\frac{1}{4} \eta^{\lambda \rho} K(x), \\
G_{\sigma \mu}(x)= & \partial_{\sigma} V_{\mu}(x)-\partial_{\mu} V_{\sigma}(x) \\
& -\operatorname{ig}\left[V_{\sigma}(x), V_{\mu}(x)\right], \\
C^{\rho}(x)= & -\frac{\mathrm{i}}{4} \partial_{\lambda} A^{\lambda \rho}(x)-\frac{1}{32} \partial^{2} V^{\rho}(x) .
\end{aligned}
$$

Before giving the final expression for $\mathcal{V}^{\rho}$, we note it may be now re-written as

$$
\begin{aligned}
\mathcal{V}^{\rho}(x, \theta)= & V^{\rho}(x)+\frac{\mathrm{i}}{\sqrt{2}} \bar{\theta} \gamma^{\rho} \chi(x)-\bar{\theta} \gamma^{5} \gamma_{\lambda} \theta A^{\lambda \rho}(x) \\
& -\bar{\theta} \gamma^{5} \theta \bar{\theta} B^{\rho}(x)-\left[-\frac{\mathrm{i}}{4} \bar{\theta} \gamma^{5} \gamma^{\mu} \theta \partial_{\mu}\right] \bar{\theta} \gamma^{5} \gamma_{\lambda} \theta \\
& \times\left(A^{\lambda \rho}(x)-\frac{\mathrm{i}}{8} \partial^{\lambda} V^{\rho}\right),
\end{aligned}
$$

since $\bar{\theta} \gamma^{5} \gamma^{\mu} \theta \bar{\theta} \gamma^{5} \gamma^{\lambda} \theta=\eta^{\mu \lambda}\left(\bar{\theta} \gamma^{5} \theta\right)^{2}$, hence

$$
\begin{aligned}
& \mathcal{V}^{\rho}(x, \theta) \\
& =\exp \left[-\frac{\mathrm{i}}{4} \bar{\theta} \gamma^{5} \gamma^{\mu} \theta \partial_{\mu}\right] \\
& \times\left[V^{\rho}(x)+\frac{\mathrm{i}}{\sqrt{2}} \bar{\theta} \gamma^{\rho} \chi(x)\right. \\
& \quad-\bar{\theta} \gamma^{5} \gamma_{\lambda} \theta\left(A^{\lambda \rho}(x)-\frac{\mathrm{i}}{4} \partial^{\lambda} V^{\rho}(x)\right) \\
& \left.-\bar{\theta} \gamma^{5} \theta \bar{\theta}\left(B^{\rho}(x)+\frac{1}{4 \sqrt{2}} \gamma^{\mu} \gamma^{\rho} \partial_{\mu} \chi(x)\right)\right],
\end{aligned}
$$

as is easily checked, where note that the quadratic term $\left(-\mathrm{i} \bar{\theta} \gamma^{5} \gamma^{\mu} \theta \partial_{\mu} / 4\right)^{2} / 2$ from the exponential generates also a term $-\partial^{2} V^{\rho} / 32$ from the $\theta$-independent term $V^{\rho}$ within the square brackets in Equation (23). Since the exponential term represents the translation operator of the argument $x^{\mu}$ of the component fields by $-\mathrm{i} \bar{\theta} \gamma^{5} \gamma^{\mu} \theta$, our final expression for $\mathcal{V}^{\rho}(x, \theta)$, in the Wess-Zumino supergauge, becomes simply

$$
\begin{aligned}
& \mathcal{V}^{\rho}(x, \theta) \\
&= V^{\rho}(\hat{x})+\frac{\mathrm{i}}{\sqrt{2}} \bar{\theta} \gamma^{\rho} \chi(\hat{x}) \\
&+\bar{\theta} \gamma^{5} \gamma_{\lambda} \theta\left[\frac{\mathrm{i}}{4} G^{\lambda \rho}(\hat{x})+\frac{1}{8} \varepsilon^{\rho \sigma \mu \lambda} G_{\sigma \mu}(\hat{x})-\frac{1}{4} \eta^{\lambda \rho} K(\hat{x})\right] \\
&+\bar{\theta} \gamma^{5} \theta \bar{\theta}\left[\frac{1}{2 \sqrt{2}} \gamma^{\rho} \gamma^{\sigma} \frac{1-\gamma^{5}}{2}\right. \\
&\left(\partial_{\sigma} \chi(\hat{x})-\operatorname{ig}\left(V_{\sigma}(\hat{x}) \chi(\hat{x})-\chi(\hat{x}) V_{\sigma}(\hat{x})\right)\right], \\
& \hat{x}^{\mu}=x^{\mu}-\frac{\mathrm{i}}{4} \bar{\theta} \gamma^{5} \gamma^{\mu} \theta .
\end{aligned}
$$

Using the relation $\mathcal{V}^{\rho}=t_{\alpha} \mathcal{V}_{\alpha}^{\rho}$, with the (Hermitian) matrices $t_{\alpha}$ as the generators of the underlying group, Equation (24) is equivalently expressed as

$$
\begin{aligned}
& \mathcal{V}_{\alpha}^{\rho}(x, \theta) \\
= & V_{\alpha}^{\rho}(\hat{x})+\frac{\mathrm{i}}{\sqrt{2}} \bar{\theta} \gamma^{\rho} \chi_{\alpha}(\hat{x}) \\
& +\bar{\theta} \gamma^{5} \gamma_{\lambda} \theta\left[\frac{\mathrm{i}}{4} G_{\alpha}^{\lambda \rho}(\hat{x})+\frac{1}{8} \varepsilon^{\rho \sigma \mu \lambda} G_{\alpha \sigma \mu}(\hat{x})-\frac{1}{4} \eta^{\lambda \rho} K_{\alpha}(\hat{x})\right] \\
& +\bar{\theta} \gamma^{5} \theta \bar{\theta}\left[\frac{1}{2 \sqrt{2}} \gamma^{\rho} \gamma^{\sigma} \frac{1-\gamma^{5}}{2}\left(\nabla_{\sigma} \chi(\hat{x})\right)_{\alpha}\right],
\end{aligned}
$$

where

$$
\begin{aligned}
& G_{\alpha}^{\lambda \rho}=\partial^{\lambda} V_{\alpha}^{\rho}-\partial^{\rho} V_{\alpha}^{\lambda}+\mathrm{g} f_{\alpha \beta \gamma} V_{\beta}^{\lambda} V_{\gamma}^{\rho}, \\
& \left(\nabla^{\sigma} \chi\right)_{\alpha}=\partial^{\sigma} \chi_{\alpha}+\operatorname{ig}\left(T_{\beta}\right)_{\alpha \gamma} V_{\beta}^{\sigma} \chi_{\gamma},
\end{aligned}
$$

and $T_{\beta}$ denotes the adjoint representation of the generators.

\section{Conclusion}

Although the derivation is somehow tedious, the final expression of the pure vector superfield and its theta dependent part are not complicated. The explicit expression for the pure vector superfield allows the construction of the supersymmetric action corresponding directly to the so-called $\mathcal{D}$-term, as is readily checked, rather than from the $\mathcal{F}$-term constructed out of a spinor superfield, as is usually done. We hope that this novel expression of 
a pure vector superfield, derived here, will be useful in supersymmetric (vector) gauge theories and justifies this analysis.

\section{Acknowledgements}

The author would like to thank his colleagues at the Institute for the interest they have shown in this work.

\section{REFERENCES}

[1] P. Binétruy, "Supersymmetry: Theory, Experiment, and Cosmology,” Oxford University Press, Oxford, 2006.

[2] H. Baer and X. Tata, "Weak Scale Supersymmetry: From
Superfields to Scattering Events,” Cambridge University Press, Cambridge, 2006.

doi:10.1017/CBO9780511617270

[3] S. Weinberg, "The Quantum Theory of Fields, Vol. III, Supersymmetry," Cambridge University Press, Cambridge, 2000.

[4] S. Ferrara (Editor), "Supersymmetry," North-Holland, Amsterdam, 1987.

[5] A. Salam and J. Strathdee, "Superfields and Fermi-Bose Symmetry,” Physical Review D, Vol. 11, No. 6, 1975, pp. 1521-1535. doi:10.1103/PhysRevD.11.1521

[6] A. Salam and J. Strathdee, "Supersymmetry and Nonabelian Gauges,” Physics Letters B, Vol. 51, No. 4, 1974, pp. 353-355. 